Int. J. Morphol.,

32(2):703-708, 2014

\title{
Estimación y Comparación de la Masa Muscular por Segmento, en Deportistas Juveniles Chilenos
}

\author{
Muscle Mass Estimation and Comparison by Segment, in Young Chilean Athletes \\ Fernando Javier Rodríguez Rodríguez*; Héctor Ismael González Fuenzalida*; José Luis Cordero Ortiz*; \\ Sebastián Lagos Nieto*; Rodrigo Antonio Aguilera Tapia* \& Fernando Omar Barraza Gómez*
}

RODRÍGUEZ, R. F. J.; GONZÁLEZ, F. H. I.; CORDERO, O. J. L.; LAGOS, N. S.; AGUILERA, T. R. A. \& BARRAZA, G. F. O. Estimación y comparación de la masa muscular por segmento, en deportistas juveniles chilenos. Int. J. Morphol., 32(2):703-708, 2014.

RESUMEN: El objetivo de este trabajo fue determinar y comparar la composición corporal de deportistas chilenos juveniles destacados, pertenecientes a las selecciones nacionales en el año 2011, de diferentes disciplinas deportivas mediante métodos antropométricos. Fueron evaluados 4 grupos de deportistas juveniles de alto rendimiento, con un número total de 92 individuos, pertenecientes a las disciplinas de lucha olímpica, halterofilia, básquetbol y fútbol. La Masa Muscular de los miembros superiores (MMES) es mayor en basquetbolistas y Halterofilistas con 7,49 $( \pm 1,25)$ y 7,50 $( \pm 2,54) \mathrm{kg}$ respectivamente, al ser comparado con futbolistas, quienes obtuvieron un promedio de 5,27 ( $\pm 0,37) \mathrm{kg}$. Por otra parte la lucha olímpica posee un promedio de masa muscular de 6,46 ( $\pm 1,50) \mathrm{kg}, 1 \mathrm{a}$ cual está por debajo de los basquetbolistas y Halterofilistas, debido a que la utilización de los miembros superiores es menor que en las disciplinas anteriormente mencionadas, pero a su vez, es mayor que la de los futbolistas. No se aprecian diferencias importantes en la masa muscular total, pero que son marcadas en la masa muscular por segmentos. Concluimos que la estimación de la masa muscular total, no es un elemento suficiente para determinar y predecir el rendimiento deportivo de los sujetos, a diferencia de la segmentación de la masa muscular que permite comparar de mejor forma, la manera en que se distribuye este componente en las distintas disciplinas deportivas, mejorando la especificidad del conocimiento.

PALABRAS CLAVE: Composición corporal; Masa muscular total; Masa muscular por segmentos.

\section{INTRODUCCIÓN}

Cada especialidad deportiva tiene definido un patrón de cineantropometría específico (Villa et al., 1992), en el cual las características físicas están ligadas al rendimiento deportivo y a la asociación que se puede establecer entre la distribución de los componentes morfoestructurales (grasa, músculo, hueso, etc) (Barraza et al., 2009).

Los métodos de determinación de la composición corporal existentes en la actualidad permiten determinan el valor total de los componentes corporales, sean estos de masa adiposa, muscular, ósea, residual y piel (Matiegka, 1921; Behnke, 1942; Brozek et al., 1963; Siri, 1961; Parizkova et al., 1971; Würch, 1974; Rocha, 1975; Jackson \& Pollock, 1978; Lohman, 1981; Drinkwater, 1984a; Kerr, 1988), pero no definen la distribución de los componentes en el cuerpo.

La masa muscular es uno de los componentes que mejor se relaciona con el rendimiento deportivo, por lo tanto su determinación en fundamental (Carter, 1984).
Donald Drinkwater en su tesis doctoral, presenta un protocolo de separación de los componentes corporales (Grasa, musculo, tejido residual y óseo) por medio de ecuaciones de regresión, reconociendo a los miembros y tronco como cilindros y conos geométricos, obteniendo una idea de cada componente por región anatómica (Drinkwater et al., 1984b; Drinwater), concepto que varios autores rechazan.

Es posible estimar la masa muscular total por medio de ecuaciones antropométricas, a partir de Densitometría Fotónica Dual (DEXA) (Visser et al., 1999; Shih et al., 2000; Levine et al., 2000; Kim et al., 2002, 2004), pero no se ha dividido este componente por segmentos corporales.

En un estudio realizado en deportistas recreativos, se pudieron crear fórmulas antropométricas a partir de ecuaciones de regresión, por medio del DEXA, para determinar la distribución corporal de la masa muscular (Rodríguez et al., 2010a). Este método permite obtener información importante de la 
distribución de la masa muscular, la cual se divide en masa muscular de los miembros superiores (MMES) y masa muscular de miembros inferiores (MMEI). De esta manera es posible conocer cómo se distribuye este componente fundamental para el desarrollo de la actividad física y que cambia según la disciplina deportiva que se realiza.

El objetivo de este trabajo fue determinar y comparar la composición corporal de deportistas chilenos juveniles destacados, pertenecientes a las selecciones nacionales en el año 2011, de diferentes disciplinas deportivas mediante métodos antropométricos. Además la misma muestra fue utilizada para determinar y comparar la distribución de la masa muscular por segmentos, con el fin de poder explicar de qué manera influyen las exigencias individuales de cada deporte estudiado y como afectan éstas, a la distribución de la masa muscular.

\section{MATERIAL Y MÉTODO}

Se evaluaron 4 grupos de deportistas seleccionados juveniles de alto rendimiento, un total de 92 individuos, pertenecientes a las disciplinas de lucha olímpica, halterofilia, básquetbol y fútbol.

La selección nacional de lucha olímpica estaba constituida por un total de 25 deportistas cuya edad promedio fue 19 ( $\pm 3,32)$. Los seleccionados nacionales y regionales de halterofilia fueron un total de 13 deportistas cuya edad promedio fue $17,46( \pm 3,45)$. La selección nacional de basquetbol estaba conformada por un total de 12 jugadores, con una edad promedio de $16,33( \pm 0,65)$ y los jugadores de fútbol de las categorías juveniles del año 2007 pertenecientes al Club Everton de Viña del Mar, estaba conformado por 42 deportistas, con una edad promedio de $17,10( \pm 0,98)$. Todos evaluados bajo un consentimiento informado de los procesos y uso de los datos de la investigación.

Las evaluaciones fueron realizadas en el Centro de Entrenamiento Olímpico (CEO) Centro de Entrenamiento Regional (CER) en Santiago, para las disciplinas incluidas en esta evaluación. Las evaluaciones se realizaron durante la mañana, en ayuno y después del primer vaciado vesical.

El proceso antropométrico se realizó con un Kit Gaucho Pro "Mercosur", fabricado en Argentina bajo licencia de Rosscraft Canadá para su comercialización en el Continente Americano.

El kit antropométrico está compuesto por los instrumentos: Campbell 20 (Antropómetro largo), Campbell 10 (antropómetro corto), segmómetro, escuadra, calibrador de pliegues cutáneos, cinta métrica para perímetros, El peso y la estatura se evalúan con la balanza Seca 700 con estadiómetro incorporado.

La evaluación antropométrica se realizó bajo el protocolo de marcaje de la International Society for the Avancement of Kineatropometry (ISAK) y de evaluación descritos por Donald Drinkwater (Drinkwater) para el protocolo de completo de variables. La recolección de los datos se facilita por el asistente que ayuda al evaluador a registrar los datos, dicho asistente conocía las técnicas de medición lo que permitió un trabajo fluido, ya que aseguraba la secuencia correcta de los lugares de medición.

Se evaluaron 3 variables básicas, peso, estatura de pié y estatura sentado, 9 diámetros óseos, 14 perímetros, 8 pliegues cutáneos, y 8 longitudes. Los datos fueron recolectados en una planilla en papel y posteriormente transcritos a una planilla Excel.

La composición corporal de los individuos fue determinada a través del Programa de cineantropometría Cine Gim 2002 versión 1.8 creado por el Lic. José Luis Arcodia.

La masa muscular por segmento fue determinada a través de las formulas creadas por Rodríguez et al. (2010a). Masa muscular de los miembros superiores $(\mathrm{MMES})=[(\mathrm{T}-\mathrm{PLT}) *$ $(0,043 * \mathrm{PBR} 2)]-\mathrm{P} / 1000$

Donde, T: Talla; PLT: Pliegue Tricipital; PBR: Perímetro Brazo Relajado; P: Peso Corporal.

Para la estimación de masa muscular de los miembros inferiores $(\mathrm{MMEI})=[(\mathrm{T}-\mathrm{SPMP}) * \mathrm{DF} 2]+(\mathrm{PMM}+\mathrm{PP}) /$ 1000. Donde, T: Talla; SPMP: Sumatoria de Pliegues del Muslo medio y pierna; DF: Diámetro Femoral; PMM: Perímetro Muslo Medio; PP: Perímetro de la Pierna.

Los datos fueron registrados en planillas de Microsoft Excel de Office 2007. Los resultados son analizados en la plataforma SPSS versión $\mathrm{n}^{\mathrm{o}} 17$ para obtención de significancia en una prueba T-Student, considerando un nivel significativo cuando $\mathrm{p}<0,05$.

\section{RESULTADOS}

En los grupos de jóvenes deportistas seleccionados, el promedio de edad de la Lucha Olímpica supera a las otras tres disciplinas, las cuales son muy similares entre sí (Tabla I). La prueba T-Student arroja un valor entre la edad de Lucha y Básquetbol de p<0,0048 y Lucha y Fútbol p<0,000. 
En cuanto al peso se aprecia que los resultados más altos corresponden a la disciplina del básquetbol, seguido de la Halterofilia y siendo el Fútbol quien posee los valores más bajos (Tabla I). La prueba T-Student entre Halterofilia y Fútbol p<0,001, Lucha y Fútbol $p<0,003$, Básquetbol y Fútbol $\mathrm{p}<0,000$.

La talla de los deportistas es mayor en los jugadores de básquetbol, menor en los pesistas $(\mathrm{p}<0,000)$ y encontrándose en segundo y tercer lugar con una mínima diferencia, la lucha $(\mathrm{p}<0,000)$ y fútbol $(\mathrm{p}<0,000)$ siendo este último levemente más alto (Tabla I).

La masa muscular de los deportistas evaluados tiene valores muy similares entre Halterofilia y Básquetbol, siendo este el último el más alto. El valor más bajo lo obtuvo el Fútbol (Tabla II). Los valores obtenidos son, Halterofilia y Fútbol ( $p<0,000)$, Lucha y Fútbol ( $p<0,000)$, Básquetbol y Fútbol $(\mathrm{p}<0,000)$.

En el Básquetbol se encuentran los valores más altos en cuanto a la masa adiposa seguida por la Halterofilia y Fútbol (Tabla II), obteniendo el valor más bajo la disciplina de Lucha Olímpica $(\mathrm{p}<0,02)$.
La masa ósea posee valores muy similares entre las cuatro disciplinas, teniendo el valor más alto el básquetbol y el fútbol el más bajo (Tabla II). Halterofilia y Fútbol $(\mathrm{p}<0,009)$, Lucha Olímpica y Fútbol $(\mathrm{p}<0,007)$, Básquetbol y Fútbol $(\mathrm{p}<0,000)$.

En cuanto a la masa residual en el Básquetbol encontramos los valores más altos y en Fútbol los valores más bajos (Tabla II). Halterofilia y Fútbol $(\mathrm{p}<0,005)$, Lucha y Fútbol ( $p<0,01)$, Básquetbol y Fútbol $(\mathrm{p}<0,000)$. La misma relación ocurre al observar los datos de la masa piel. Básquetbol y Halterofilia $(\mathrm{p}<0,02)$, y Básquetbol y Fútbol $(\mathrm{p}<0,000)$.

Masa muscular en kilogramos de los miembros apendiculares (Tabla III).

La masa muscular de los miembros superiores es baja en el grupo de Lucha Olímpica y en Futbol ( $\mathrm{p}<0,000)$, igualmente en la masa muscular del tronco donde presenta los valores más bajos que el resto de los grupos de deportistas $(\mathrm{p}<0,002)$. La masa muscular de los miembros superiores es mayor en el grupo de Basquetbol, que en el resto de los deportistas con un $\mathrm{p}<0,04$.

Tabla I. Promedio y desviación estándar (D.E) de los datos básicos determinados en los deportistas seleccionados juveniles de alto rendimiento.

\begin{tabular}{lcccccc}
\hline & Edad (años) & (D.E.) & Peso $(\mathbf{K g})$ & (D.E.) & \multicolumn{1}{c}{ Talla (cm) } & (D.E.) \\
\hline Halterofilia & 17,46 & $\pm 3,45$ & 77,16 & $\pm 23,25$ & 166,44 & $\pm 7,09$ \\
Lucha Olímpica & ${ }^{(1)} 19,00$ & $\pm 3,32$ & 72,28 & $\pm 15,09$ & 169,33 & $\pm 6,73$ \\
Básquetbol & 16,33 & $\pm 0,65$ & 80,43 & $\pm 10,39$ & (3) 186,71 & $\pm 8,93$ \\
Fútbol & 17,10 & $\pm 0,98$ & (2) 65,00 & $\pm 5,63$ & 170,05 & $\pm 5,44$ \\
\hline
\end{tabular}

(1) Diferencias significativas en una Prueba T-Student con un valor de $\mathrm{p}<0,05$ entre la edad de lucha y básquetbol.

(2) Diferencias significativas en una Prueba T-Student con un valor de $p<0,05$ entre el peso de fútbol y el resto de los deportistas.

(3) Diferencias significativas en una Prueba T-Student con un valor de $\mathrm{p}<0,05$ entre la talla de básquetbol y los demás deportistas.

Tabla II. Composición corporal en los en los deportistas seleccionados juveniles de alto rendimiento evaluados.

\begin{tabular}{lrrrrrrrr}
\hline & \multicolumn{2}{c}{ Halterofilia } & \multicolumn{2}{c}{ Lucha Olímpica } & \multicolumn{2}{c}{ Basquetbol } & \multicolumn{2}{c}{ Fútbol } \\
& \multicolumn{1}{c}{ Kg } & \multicolumn{1}{c}{ (D.E) } & \multicolumn{1}{c}{ Kg } & \multicolumn{1}{c}{ D.E } & \multicolumn{1}{c}{ Kg } & \multicolumn{1}{c}{ D.E. } & \multicolumn{1}{c}{ Kg } & D.E \\
\hline Masa muscular & 40,48 & $\pm 12,46$ & 36,84 & $\pm 7,80$ & 40,50 & $\pm 7,15$ & ${ }^{(1)} 32,18$ & $\pm 3,32$ \\
Masa grasa & 15,79 & $\pm 6,75$ & 14,38 & $\pm 5,86$ & ${ }^{(2)} 16,80$ & $\pm 3,48$ & 14,89 & $\pm 2,87$ \\
Masa ósea & 8,85 & $\pm 2,77$ & 8,51 & $\pm 1,31$ & 9,28 & $\pm 0,99$ & ${ }^{(3)} 7,93$ & $\pm 0,78$ \\
Masa residual & 9,24 & $\pm 3,42$ & 8,62 & $\pm 2,69$ & 9,71 & $\pm 1,40$ & ${ }^{(4)} 7,67$ & $\pm 0,94$ \\
Masa piel & 3,77 & $\pm 0,57$ & 3,78 & $\pm 0,71$ & ${ }^{(5)} 4,25$ & $\pm 0,34$ & 3,62 & $\pm 0,20$ \\
\hline
\end{tabular}

(1) Diferencias significativas en una "Prueba T" entre la masa muscular del grupo de Futbol y el resto de los deportistas.

(2) Diferencias significativas en una "Prueba T" entre la masa grasa del básquetbol y lucha olímpica.

(3) Diferencias significativas en una "Prueba T" entre la masa ósea del equipo de Fútbol y el resto de los deportistas.

(4) Diferencias significativas en una "Prueba T" entre la masa residual del equipo de fútbol y el resto de los deportistas.

(5) Diferencias significativas en una "Prueba T" entre la masa piel del grupo de básquetbol y halterofilia y entre básquetbol y fútbol. 
Tabla III. Masa muscular en kilogramos apendicular y axial de las disciplinas evaluadas.

\begin{tabular}{lcccc}
\hline & MMES(Kg) & D.E. & MMEI (Kg) & (D.E.) \\
\hline Halterofilia & 7,50 & $\pm 2,54$ & 15,76 & $\pm 5,11$ \\
Lucha Olímpica & ${ }^{(1)} 6,46$ & $\pm 1,50$ & 15,17 & $\pm 2,10$ \\
Basquetbol & 7,49 & $\pm 1,25$ & ${ }^{(3)} 19,06$ & $\pm 2,85$ \\
Fútbol & ${ }^{(2)} 5,27$ & $\pm 0,37$ & 16,25 & $\pm 1,59$ \\
\hline
\end{tabular}

(1) Diferencias significativas en una "Prueba T" entre masa muscular miembros superiores de la lucha olímpica y halterofilia y lucha olímpica y básquetbol.

(2) Diferencias significativas en una "Prueba T" entre masa muscular de los miembros superiores del grupo de fútbol y el resto de los deportistas.

(3) Diferencias significativas en una "Prueba T" entre masa muscular miembros inferiores del grupo de básquetbol y el resto de los deportistas.

\section{DISCUSIÓN}

La distribución de la masa muscular por segmentos en deportistas, nos permitirá comprender de qué manera la musculatura es predominante en cada disciplina estudiada.

La MMES es mayor en basquetbolistas y halterofilistas con 7,49 $( \pm 1,25)$ y $7,50( \pm 2,54) \mathrm{kg}$ respectivamente, al ser comparado con futbolistas, quienes obtuvieron un promedio de $5,27( \pm 0,37) \mathrm{kg}$. Creemos que estas diferencias se deben a la utilización de los miembros superiores en las prácticas deportivas del básquetbol y halterofilia. En el caso de los basquetbolistas se debe a que la fuerza de los brazos es importante en el control de los rebotes (Bonafonte,1988). En cuanto a la halterofilia, sostener de manera isométrica grandes cargas de peso estimula un desarrollo de la musculatura en los miembros superiores, como señala Baechle (Baechle \& Earle, 2000), el entrenamiento de fuerza e hipertrofia producen adaptaciones que conllevan a un aumento de la masa muscular.

Por otra parte la lucha olímpica posee un promedio de masa muscular de $6,46( \pm 1,50) \mathrm{kg}$, la cual está por debajo de los basquetbolistas y halterofilistas, debido a que la utilización de los miembros superiores es menor que en las disciplinas anteriormente mencionadas, pero a su vez, es mayor que la de los futbolistas.

Diferentes estudios han descrito a la lucha olímpica, como una especialidad de carácter intermitente, que requiere la optimización de los niveles de fuerza y potencia máxima del miembro inferior y superior (Horswill, 1992), lo cual permite comprender que la distribución de la masa muscular de los luchadores, es más equilibrada que en las otras tres disciplinas estudiadas, por lo que se justifica que no posea valores más altos, como es el caso del básquetbol y halterofilia.

Al comparar la MMES de las cuatro disciplinas, con los valores del PHANTOM, podemos observar que todas las disciplinas poseen un mayor desarrollo muscular, debido a que realizan actividad física, lo cual implica un desarrollo muscular en la totalidad del cuerpo (Tabla IV).
Tabla IV. Comparación de los resultados de la MMES y de la MMEI de los deportistas evaluados, de la muestra CHIREF y del PHANTOM.

\begin{tabular}{lcc}
\hline & MMES $(\mathbf{K g})$ & MMEI $(\mathbf{K g})$ \\
\hline Halterofilia & 7,50 & 15,76 \\
Lucha olímpica & 6,46 & 15,17 \\
Básquetbol & 7,49 & 19,06 \\
Fútbol & 5,27 & 16,25 \\
CHI REF & 6,12 & 16,56 \\
PHANTOM & 4,46 & 15,57 \\
\hline
\end{tabular}

Si se compara la muestra evaluada con una muestra denominada CHIREF, la cual tiene la finalidad de ser una referencia de composición corporal y somatotipo de jóvenes chilenos saludables entre 20 y 29 años (Rodríguez et al., 2010b). La Tabla IV muestra que CHIREF posee una MMES que sólo supera los valores del fútbol,

Comparando los resultados podemos notar un desarrollo mucho menor de la masa muscular de los futbolistas, esto es atribuible al sistema energético predominante del deporte. Según la Dra. Carniero (Carniero, 2009) el futbol es una disciplina que compromete eminentemente los procesos aeróbicos por sobre los demás, afirmando que el aporte energético a través de esta vía metabólica es de aproximadamente del $75 \%$ del máximo consumo de oxígeno, lo que podría desfavorecer el crecimiento y desarrollo de la masa muscular, ya que como nos señala Baechle, el entrenamiento aeróbico disminuye la concentración de la encima glagolítica y el volumen de las fibras de tipo II hipertrofiadas y no hipertrofiadas (Baechle \& Earle).

La masa muscular de los miembros inferiores en los deportistas, aumenta dependiendo del tipo de entrenamiento que requiere cada disciplina deportiva.

El PHANTOM con 15,57 kg de MMEI es levemente superior a la de los luchadores, quienes son superados ampliamente por el básquetbol, que posee los valores más altos. La fuerza y la potencia de piernas determinan como se realizan las acciones explosivas en baloncesto. Así, el rebote, los saltos, la velocidad y la agilidad para acelerar y cambiar rápidamente de dirección, se mejoran por el aumento de la fuerza. La carrera y lossaltos continuos que requiere 
el baloncesto, resulta en una mejora de la resistencia muscular de los miembros inferiores (Bonafonte), lo que implica un aumento en la masa muscular (Baechle \& Earle). Además la intensidad de su realización, es mayoritariamente máxima o submáxima (Gómez et al., 2007). El fútbol y la halterofilia, que siendo inferiores al básquetbol poseen valores muy similares al PHANTOM.

CHIREF posee una MMEI, que supera a todas las disciplinas excepto al básquetbol. Si consideramos que CHIREF es el promedio de la masa muscular de miembros inferiores de chilenos adultos activos, podemos decir que dicha superioridad se debe al desarrollo muscular propio de la edad (20 a 30 años).

En el caso del básquetbol, la diferencia se debe a que los deportistas evaluados poseían el mayor promedio de altura $1,87 \mathrm{mt}( \pm 8,93)$, por lo que se justifica la mayor cantidad de MMEI. Dicha aseveración se justifica con lo planteado por Spenst et al. (1993), el cual manifiesta que desde la perspectiva de un modelo físico, la masa muscular podría considerarse un cilindro cuyo diámetro está determinado por la circunferencia de los miembros y su altura por la estatura. Luego, si mantenemos constante el diámetro pero aumentamos la longitud del cilindro (talla), se incrementará su volu- men y, consecuentemente, la masa muscular, por lo que se evidencia una estrecha relación entre estatura y kilos de masa muscular.

Concluimos que la estimación de la masa muscular total, no es un elemento suficiente para determinar y predecir el rendimiento deportivo de los sujetos, a diferencia de la segmentación de la masa muscular que permite comparar de mejor forma, la manera en que se distribuye este componente en las distintas disciplinas deportivas, mejorando la especificidad del conocimiento.

\section{AGRADECIMIENTOS.}

A los entrenadores de las selecciones nacionales deportivas del CEO y CER, por su disposición y comprensión.

A la Escuela de Educación Física de la Pontificia Universidad Católica de Valparaíso, a todos y cada uno de los voluntarios evaluados y en especial a la Dirección Investigación e Innovación (DII) de la Pontificia Universidad Católica de Valparaíso, Chile por su constante apoyo.

RODRÍGUEZ, R. F. J.; GONZÁLEZ, F. H. I.; CORDERO, O. J. L.; LAGOS, N. S.; AGUILERA, T. R. A. \& BARRAZA, G. F. O. Muscle mass estimation and comparison by segment, in young Chilean athletes. Int. J. Morphol., 32(2):703-708, 2014.

SUMMARY: The aim of this study was to determine and compare by anthropometric methods, the body composition of elite young Chilean athletes, who were part of the 2011 national team in different sports. Four groups of elite young athletes were evaluated, with a total number of 92 individuals belonging to the wrestling, weightlifting, basketball and football disciplines. The muscle mass of the upper extremities (MMES) is higher in basketball players and bodybuilders with $7.49( \pm 1.25)$ and $7.50( \pm 2.54) \mathrm{kg}$ respectively, when compared with players who scored an average of $5.27( \pm 0.37) \mathrm{kg}$. Moreover, wrestling has an average muscle mass of $6.46( \pm 1.50) \mathrm{kg}$ which is below that of basketball players and bodybuilders, because the use of upper limbs is less than in disciplines mentioned above, but in turn, is greater than that of football players. No significant differences in total muscle mass are reported, however, there are marked differences in muscle mass segment. We concluded that the estimate of total muscle mass is not sufficient basis to determine and predict athletic performance of the subjects. Muscle mass segment on the other hand, provides a better comparison range for the way in which this component is distributed among different sports disciplines, improving specific knowledge and information.

KEY WORDS: Body composition; Total muscle mass; Muscle mass segment.

\section{REFERENCIAS BIBLIOGRÁFICAS}

Baechle, T. R. \& Earle, T. W. Essentials of strength training and conditioning. $2^{\text {nd }}$ ed. Champaign, Human Kinetics, 2000.

Barraza , G. F.; Hadler, G. A.; Jeria, F. J. \& Riffo, E.C. Cuantificación de la masa muscular de los miembros apendiculares, por medio ecuaciones antropométricas. Rev. Motr. Hum., 10(2):44-8, 2009.

Behnke, A. R.; Freen, B. G. \& Welham, W. C. Specific gravity of healthy men. JAMA, 118(7):495-8, 1942.
Bonafonte, L. F. Fisiología del baloncesto. Arch. Med. Deporte, 15 (68):479-483, 1988.

Brozek, J.; Grande, F.; Anderson, J. \& Keys, A. Densitometric analysis of body composition: Revision of some quantitative assumptions. Ann. NY Acad. Sci., 110:113-40, 1963.

Carneiro, L. M. Valoración Morfofuncional de Futbolistas Selección Sub- 20 del Estado Monagas Macrociclo 2009- 2010. Tesis de Magister, Universidad Nacional Experimental de los 
Llanos centrales "Romulo Gallegos”, 2009.

Carter, J. E. L. Somatotype of Olympic Athletes from 1948 to 1976. Med. Sports Sci., 18:80-109, 1984.

Drinkwater, D. T. An anatomically derived method for he anthropometric estimation of human body composition. PH.D. Thesis, Fraser University, 1984a.

Drinkwater, D. T.; Martin, A. R.; Ross, W. D. \& Clarys, J. P. Validation by cadaver dissection of Matiegka's equations for the anthropometric estimation of anatomical body composition in human adults. In: Day J. A. P. (Ed.). Perspectives in Kinanthropometry. Champaign, Human Kinetics, 1984b. pp.221-7.

Gómez, M.; Lorenzo, A.; Ortega, E.; Sampaio, J. \& Ibañez, S. Diferencias en las estadísticas de juego entre bases, aleros y pívots en baloncesto femenino. $C C D, 2(6): 139-44,2007$.

Horswill, C. A. Applied Physiology of amateur Wrestting. Sport Med., 14(2):114-43, 1992.

Jackson, A. S. \& Pollock, M. L. Generalized equations for predicting body density of men. Br. J. Nutr., 40(3):497-504, 1978.

Kerr, D. A. An anthropometric method for the fractionation of skin, adipose, muscle, bone and residual tissue masses in males and females age 6 to 77 years. M.Sc. Thesis, Simon Fraser University, 1988.

Kim, J.; Wang, Z.; Heymsfield, S. B.; Baumgartner, R. N. \& Gallagher, D. Total-body skeletal muscle mass: estimation by a new dual-energy x-ray absorptiometry method. Am. J. Clin. Nutr., 76(2):378-83, 2002.

Kim, J.; Heshka, S.; Gallagher, D.; Kotler, D.; Mayer, L.; Albu, J.; Shen, W.; Freda P. \& Heymsfield, S. Intermuscular adipose tissue-free skeletal muscle mass: estimation by dual-energy X-ray absorptiometry in adults. J. Appl. Physiol., 97(2):655$60,2004$.

Levine, J.; Abboud, L.; Barry, M.; Reed, J.; Sheedy, P. \& Jensen, M. Measuring leg muscle and fat mass in humans: comparison of CT and dual-energy X-ray absorptiometry. J. Appl. Physiol., $88(2): 452-6,2000$.

Lohman, T. G. Skinfolds and body density and their relation to body fatness: A review. Hum. Biol., 53(2):181-225, 1981.

Matiegka, J. The testing of physical efficiency. Am. J. Phys. Anthropol., 4:223-330, 1921.

Parizkova, J.; Eiselt, E.; Sprynarova, S. \& Wachtlova, M. Body composition, aerobic capacity, and density of muscle capillaries in young and old men. J. Appl. Physiol., 31(3):323-5, 1971.
Rocha, M. S. L. Peso ósseo do brasileiro de ambos os sexos de 17 a 25 anhos. Arq. Anat. Antrop., 1:445-51, 1975.

Rodríguez, F. J.; Almagià, A. A. \& Berral, F. J. Estimación de la masa muscular de los miembros apendiculares, a partir de densitometría fotónica dual (DEXA). Int. J. Morphol., 28(4):1205-10, 2010a.

Rodríguez, R. F. J.; Almagià, F. A. A.; Yuing, F. T.; Binvignat, G. O. \& Lizana, A. P. Composicion corporal y somatotipo referencial de sujetos fisicamente activos. Int. J. Morphol., 28(4):1159-65, 2010b.

Shih, R.; Wang, Z.; Heo, M.; Wang, W. \& Heymsfield, S. B. Lower limb skeletal muscle mass: development of dual-energy X-ray absorptiometry prediction model. J. Appl. Physiol., 89(4):13806,2000 .

Siri, W. E. Body Composition from fluid space and density. Analysis of Methods. En: Techniques of Measuring Body Composition. En: Brozek, J. \& Henshcel, A. (Eds.) Techniques for measuring body composition. Washington DC, National Academy Press, 1961. pp.223-44.

Spenst, L. F.; Martin, A. D. \& Drinkwater, D. T. Muscle mass of competitive male athletes. J. Sport Sci., 11(1):3-8, 1993.

Villa, J. G.; De Paz, J. A. \& González-Gallego, J. Bases para la evaluación de la condición física y la preparación deportiva. En: Santonja, R. (Ed.). Libro Olímpico de Medicina Deportiva. Madrid, C.O.E., 1992. pp.23-34.

Visser, M.; Fuerst, T.; Lang, T.; Salamone, L. \& Harris T. Validity of fan-beam dualenergy X-ray absorptiometry for measuring fat-free mass and leg muscle mass J. Appl. Physiol., 87(4):1513-20, 1999.

Würch, A. La femme et le sport. Med. Sport Française, 4(1):4415,1974 .

\author{
Dirección para correspondencia: \\ Fernando Rodríguez Rodríguez \\ Avda. El Bosque 1290 \\ Casilla 4059 \\ Valparaíso \\ Pontificia Universidad Católica de Valparaíso \\ CHILE
}

E-mail: fernando.rodriguez@ucv.cl

Recibido : 01-01-2013

Aceptado: 22-03-2014 